\title{
Aclaramiento de lactato como predictor de mortalidad en pacientes con choque séptico
}

\section{Lactate clearance as a predictor of mortality in septic shock patients}

Recibido: $18 / 06 / 2021$

Aceptado: 22/07/2021

\author{
Mahobeny Natalia Méndez Mejía \\ Universidad de San Carlos de Guatemala \\ mendezmahobeny@gmail.com \\ https://orcid.org/0000-0002-9240-8040
}

\section{Referencia del artículo}

Méndez Mejía, M. N. (2021). Aclaramiento de lactato como predictor de mortalidad en pacientes con choque. Revista Diversidad Científica, 1(1), 1-7. DOl: https://doi.org/10.36314/diversidad.v1i1.1

\section{Resumen}

La sepsis es una de las principales causas de muerte en las unidades de cuidados intensivos pediátricos. El tratamiento en los pacientes con diagnóstico de choque séptico es dirigido a alcanzar metas tempranas en los estadíos primarios de la presentación de la enfermedad. Se realizó revisión y lectura de material científico. El aclaramiento de lactato a las 24 horas después de iniciada la terapia de reanimación demuestra ser el mejor predictor de mortalidad a corto plazo en los pacientes que presentan choque séptico. La hiperlactatemia diagnosticada al ingreso a las unidades de terapia intensiva también se relaciona con mayor frecuencia de lesión renal aguda, mayor tiempo de ventilación mecánica, necesidad de dosis más altas de aminas vasoactivas y aumento de la estancia hospitalaria. Las concentraciones de lactato venoso se consideran un biomarcador de mayor utilidad que los valores de lactato arterial para predecir mortalidad en estos pacientes. Se concluye que los niveles de lactato superiores a tres milimoles/litro y un aclaramiento de lactato menor al $10 \%$ posterior a la terapia de reanimación inicial se encuentra asociado con un aumento de la mortalidad.

Palabras clave: aclaramiento de lactato, choque séptico, mortalidad 


\begin{abstract}
Sepsis is one of the leading causes of death in pediatric intensive care units. Treatment in patients with a diagnosis of septic shock is aimed at achieving early goals in the primary stages of the presentation of the disease. Review and reading of scientific material was carried out. Lactate clearance 24 hours after resuscitation therapy has been shown to be the best predictor of short-term mortality in patients with septic shock. Hyperlactatemia diagnosed on admission to intensive care units is also associated with a higher frequency of acute kidney injury, longer time on mechanical ventilation, the need for higher doses of vasoactive amines, and an increase in hospital stay. Venous lactate concentrations are considered a more useful biomarker than arterial lactate values to predict mortality in these patients. It is concluded that lactate levels greater than three millimoles / liter and a lactate clearance less than $10 \%$ after initial resuscitation therapy is associated with an increase in mortality.
\end{abstract}

Keywords: lactate clearance, septic shock, mortality 


\section{Revista Diversidad Científica Vol. 1 No. 1 Año 2021}

\section{Introducción}

La sepsis es responsable de muchas admisiones a las unidades de terapia intensiva y su progresión hacia choque séptico se relaciona con una tasa de mortalidad elevada, haciéndose necesario reconocer de forma precoz esta patología para evitar la transición hacia el choque séptico que causa muchas muertes anualmente.

El ácido láctico se descubrió en 1780, y desde entonces se han realizado diversos estudios para demostrar la utilidad de éste como biomarcador en diferentes patologías. Se considera hiperlactatemia cuando los valores de lactato venoso o arterial son superiores a dos milimoles/litro. Los pacientes con choque séptico utilizan el metabolismo anaeróbico, para satisfacer las demandas energéticas necesarias, esto también genera lactato que al acumularse en los tejidos produce acidosis metabólica. Los valores de lactato sérico se utilizan como marcador de la perfusión tisular.

Se denomina aclaramiento de lactato, a la disminución de la concentración de lactato a través del tiempo. Para medir la depuración de lactato se debe obtener una muestra sanguínea al ingreso y posteriormente muestras sanguíneas horas después de la reanimación inicial. La medición del lactato se realiza como un procedimiento de rutina para conocer la evolución clínica del paciente al manejo terapéutico inicial mediante la toma de sangre para gases venosos o arteriales, ésta prueba es de mediano costo y se encuentra disponible en muchos de los hospitales del país.

Se procedió a realizar una investigación documental, cuyo objetivo fue fundamentar que el aclaramiento de lactato puede predecir el riesgo de muerte en pacientes con diagnóstico de choque séptico. Se encontró que los pacientes que presentan mediciones de lactato superiores a dos milimoles/litro al ingresar a las unidades de terapia intensiva está relacionado con mayor frecuencia de sufrir lesión renal aguda, ventilación mecánica por tiempo prolongado, necesidad de dosis más altas de aminas vasoactivas y aumenta las probabilidades de mortalidad; una depuración de lactato inferior al $10 \%$ después de administrada la terapia de reanimación inicial se relaciona con mayor riesgo de mortalidad y que las concentraciones de lactato sérico superiores a tres milimoles/litro son útiles como marcador de enfermedad grave con un alto riesgo de muerte. Por ello, es necesario utilizar el aclaramiento de lactato a las 24 horas como biomarcador para el pronóstico de muerte y conocer la respuesta tras administrar la terapia de reanimación en pacientes con choque séptico. Así como, realizar estudios clínicos prospectivos en los hospitales de la región que investiguen el lactato como indicador del pronóstico de muerte y falla multiorgánica en pacientes con choque séptico. 


\section{Contenido}

Sepsis y aclaramiento de lactato

Los procesos infecciosos provocan una respuesta en el huésped, a través de diversos mecanismos proinflamatorios y antiinflamatorios que contribuyen a eliminar la infección y al reparamiento de los tejidos, pero también ocasionan daño de algunos órganos. El tipo de respuesta depende de las propiedades del agente causal, y de ciertas características del huésped como predisposiciones genéticas y enfermedades concomitantes (Angus \& Van der Poll, 2013). Sepsis se define como "una disfunción orgánica potencialmente mortal causada por una respuesta desregulada del huésped a la infección" (Singer et al. 2016). La acidosis láctica se produce cuando el ácido láctico se acumula en el interior de las células debido a una disminución significativa de la disponibilidad de oxígeno en los tejidos, y por esta razón se considera indicador de la perfusión tisular.

En el documento de consenso para el diagnóstico y tratamiento de la sepsis grave de la SEMES (Sociedad Española de Medicina de Urgencias y Emergencias) y la SEMICYUC (Sociedad Española de Medicina Intensiva, Crítica y Unidades Coronarias) se incluye la medición del lactato en la valoración inicial del paciente grave (León, 2007).

En el choque séptico el valor del lactato, tanto sus mediciones iniciales como las seriadas, son útiles para valorar el éxito del tratamiento administrado. Se toman como valores de referencia las concentraciones de lactato inferiores a dos milimoles/litro. Un aumento en sus concentraciones de dos a cinco milimoles/litro no suele ocasionar signos ni síntomas específicos, cuando las concentraciones de lactato se elevan por encima de cinco milimoles/itro inician manifestaciones clínicas características de la acidosis láctica, las cuales pueden ser: taquicardia, taquipnea y alteración del estado mental, que puede presentarse desde un leve estado confusional hasta la perdida de la conciencia. La respiración generalmente es profunda y rápida (respiración de Kussmaul) como respuesta compensatoria a la acidosis (Avalos, 2014). La medición del lactato es útil como indicador de oxigenación tisular.

Las concentraciones de lactato superiores a cinco milimoles/litro se relacionan con un pobre pronóstico en pacientes en estado crítico. Diversos estudios demuestran elevación de los valores de lactato en cuadros clínicos relacionados con traumatismo, sepsis o en pacientes en estado crítico, y que éste además es un signo de mal pronóstico e indica la necesidad de intensificar medidas terapéuticas de forma inmediata. Si con el tratamiento instaurado se reducen las concentraciones de lac- 
tato en 24 a 48 horas, se incrementan las probabilidades de supervivencia (Guevara et al. 2010).

Los niveles elevados de lactato en sangre ponen de manifiesto la disfunción celular en la sepsis. Los valores superiores a tres milimoles/litro son un criterio para la identificación de choque séptico. Cuando el lactato sérico persiste elevado a pesar la estabilización hemodinámica del paciente (presión arterial sistólica $>90 \mathrm{mmHg} 0$ una presión arterial media $>65 \mathrm{mmHg}$ ) indica una mala perfusión en los tejidos que debería sugerir una intensificación del tratamiento (León et al. 2007).

Se conoce como aclaramiento o depuración de lactato, a la disminución en la concentración de lactato sérico durante horas después de su reanimación inicial. El resultado se interpreta con base al porcentaje obtenido. Un aclaramiento de lactato inferior $10 \%$ en las primeras horas tras el inicio de la terapia es un pronóstico de muerte (Cardinal et al. 2009). Un valor positivo indica una disminución o aclaramiento de lactato, mientras que un valor negativo indica un aumento en el lactato después de la intervención terapéutica inicial. Se ha demostrado que las mediciones en serie del lactato sérico con especial énfasis en su concentración a las 24 horas después del ingreso siguen siendo el factor más predictivo de mortalidad a corto plazo en las unidades de terapia intensiva.

\section{Conclusión}

El aclaramiento de lactato a las 24 horas, después de iniciada la terapia de reanimación, demuestra ser el mejor predictor de mortalidad a corto plazo en las unidades de cuidados intensivos. Un aclaramiento de lactato inferior al 10\% después de administrada la terapia de reanimación inicial para el shock séptico, se relaciona con aumento de la mortalidad en pacientes con choque séptico. Las concentraciones de lactato sérico superiores a tres milimoles/litro, son útiles como marcador de enfermedad grave con un alto riesgo asociado de muerte.

\section{Referencias}

Angus, D. C., \& Van der Poll, T. (2013). Severe sepsis and septic shock. The New England Journal of Medicine, 369, 840-851. 10.1056/NEJMra1208623. https:// doi.org/10.1056/NEJMra1208623 
Avalos, E. I. C. (2014). Aclaramiento de lactato indicador pronóstico de mortalidad en pacientes con sepsis severa y choque séptico [Tesis de maestría, Universidad de San Carlos de Guatemala]. Biblioteca USAC. http://biblioteca.usac.edu.gt/ tesis/05/05_9331.pdf.

Cardinal Fernández, P. A., Olano, E., Acosta, C., Bertullo, H., Albornoz, H., \& Bagnulo, $\mathrm{H}$. (2009). Valor pronóstico del aclaramiento de lactato en las primeras $6 \mathrm{~h}$ de evolución en medicina intensiva. Medicina intensiva, 33(4), 166-170. https://doi. org/10.1016/S0210-5691(09)71212-4

Guevara Ramírez, P., Díaz García, R., Galán Ortega, A., Guillén Campuzano, E., Malumbre, S., Marin Soria, J. L., Muñoz Pérez, M., Navarro Segarra, X., Oliver Sáez, P., Oujo, E., del Río Barcenilla, N., \& Buño Soto, A. (2010). Lactato: utilidad clínica y recomendaciones para su medición. https://elenfermerodelpendiente.files.wordpress.com/2015/12/n-lactato-utilidad-clc3adnica-y-recomendaciones-para-su-medicic3b3n-2010.pdf

León Gil, C., García-Castrillo Riesgo, L., Moya Mir, M., Artigas Raventós, A., Borges Sa, M., Candel González, F. J., Chanovas Borrás, M., Ferrer Roca, R., Jiménez, A. J., Loza Vázquez, A., \& Sánchez García, M. (2007). Documento de consenso (SEMES-SEMICYUC): recomendaciones del manejo diagnóstico-terapéutico inicial y multidisciplinario de la sepsis grave en los Servicios de Urgencias hospitalarios. Medicina intensiva, 31(7), 375-387. https://doi.org/10.1016/ S0210-5691(07)74842-8

Singer, M., Deutschman, C. S., Seymour, C. W., Shankar-Hari, M., Annane, D., Bauer, M., Bellomo, R., Bernard, G. R., Chiche, J. D., Coopersmith, C. M., Hotchkiss, R., S., Levy, M. M., Marshall, J. C., Martin, G., S., Opal, S. M., Rubenfeld, G., D., van der Poll, T., Vincent, J. L., \& Angus, D. C. (2016). The third international consensus definitions for sepsis and septic shock (Sepsis-3). Jama, 315(8), 801-810. https://doi.org/10.1001/jama.2016.0287

\section{Sobre la autora \\ Mahobeny Natalia Méndez Mejía}

Es Médica y Cirujana, egresada de la Universidad de San Carlos de Guatemala. La investigación de la cual escribió el articulo tiene como título "Aclaramiento de lactato como predictor de mortalidad en pacientes con choque séptico". 
Copyright (c) Mahobeny Natalia Méndez Mejía

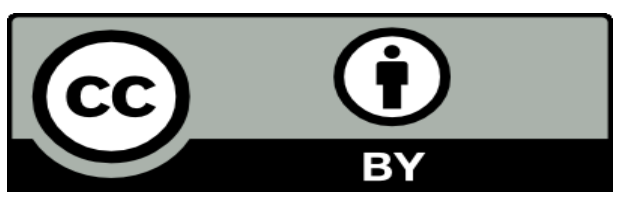

Este texto está protegido por una licencia CreativeCommons 4.0.

Usted es libre para compartir, copiar y redistribuir el material en cualquier medio o formato y adaptar el documento, remezclar, transformar y crear a partir del material para cualquier propósito, incluso comercialmente, siempre que cumpla la condición de atribución: usted debe reconocer el crédito de una obra de manera adecuada, proporcionar un enlace a la licencia, e indicar si se han realizado cambios. Puede hacerlo en cualquier forma razonable, pero no de forma tal que sugiera que tiene el apoyo del licenciante o lo recibe por el uso que hace. 